\title{
Atherosclerosis: a chronic inflammatory disease mediated by mast cells
}

\author{
PIO CONTI ${ }^{1}$, YAZDAMI SHAIK-DASTHAGIRISAEB ${ }^{2}$ \\ ${ }^{1}$ Universitŕy G. d'Annunzio, Chieti, Italy \\ ${ }^{2}$ Boston University School of Medicine, Boston, USA
}

\begin{abstract}
Inflammation is a process that plays an important role in the initiation and progression of atherosclerosis and immune disease, involving multiple cell types, including macrophages, T-lymphocytes, endothelial cells, smooth muscle cells and mast cells. The fundamental damage of atherosclerosis is the atheromatous or fibro-fatty plaque which is a lesion that causes several diseases. In atherosclerosis the innate immune response, which involves macrophages, is initiated by the arterial endothelial cells which respond to modified lipoproteins and lead to Th1 cell subset activation and generation of inflammatory cytokines and chemoattractant chemokines. Other immune cells, such as CD4+ T inflammatory cells, which play a critical role in the development and progression of atherosclerosis, and regulatory $T$ cells [Treg], which have a protective effect on the development of atherosclerosis are involved. Considerable evidence indicates that mast cells and their products play a key role in inflammation and atherosclerosis. Activated mast cells can have detrimental effects, provoking matrix degradation, apoptosis, and enhancement as well as recruitment of inflammatory cells, which actively contributes to atherosclerosis and plaque formation. Here we discuss the relationship between atherosclerosis, inflammation and mast cells.
\end{abstract}

Key words: leukocytes, inflammation, mast cells, athersclerosis.

(Cent Eur J Immunol 2015; 40 (3): 380-386)

The discovery of Toll-like receptors (TLRs) in mammalian innate immune cells at the end of the last century demonstrates once again that the innate immune response is crucial in the inflammatory process [1]. Inflammation is a process that plays an important role in the initiation and progression of atherosclerosis and circulatory diseases [2]. In fact, atherosclerosis and inflammation present similar impairment with a decrease in vasoreactivity. The fundamental lesion of atherosclerosis is the atheromatous or fibro-fatty plaque which is a lesion that causes narrowing of the artery, predisposes to thrombosis, calcifies, and causes weakening of the muscle resulting in aneurismal dilation [3]. Atherosclerosis is a chronic inflammatory and immune disease involving multiple cell types, including monocytes, macrophages, T-lymphocytes, endothelial cells, smooth muscle cells and mast cells (MCs) [4]. The importance of monocytes and macrophages in atherosclerosis was noted in the early ultra-structural studies of Still and O'Neal in 1962 [5]; while the presence of leukocytes within atherosclerotic arteries was described in the early 1980s [6]. Macrophages, however, were the first inflammatory cells to be associated with atherosclerosis. Great effort has been devoted to elucidate the molecular mechanisms by which immune cells contribute to atherosclerosis
[7]. The innate and adaptive immune systems have evolved to protect humans from pathogens, such as bacteria, virus, parasites and fungi, and the presence of infection is detected by specialized cells [8], such as macrophages, mast cells, monocytes, natural killer (NK) cells, and dendritic cells, as well as non-specialized immune cells, such as fibroblasts and others [9]. Immune-inflammatory cells of atherosclerosis can be part of innate immune response involving monocytes and macrophages that respond to the excessive uptake of lipoproteins, while adaptive immune response involves antigen-specific T cells [10]. In atherosclerosis, the innate immune response is initiated by the arterial endothelial cells which respond to modified lipoproteins and lead to the generation of inflammatory cytokines and chemoattractant chemokines [11]. Initially, researchers thought that only macrophages were present in atherosclerotic plaques, but later studies noted that other immune cells, such as CD4+ T inflammatory cells (which play a critical role in the development and progression of atherosclerosis and are the most abundant $\mathrm{T}$ cells present in atherosclerotic lesions), regulatory T cells (Treg) (which have a protective effect on the development of atherosclerosis) [12], myeloid cells (that comprise immature macrophages and granulocytes), and mast cells (which accu-

Correspondence: Pio Conti, Universitŕy G. d'Annunzio, viale Unità d'Italia, 66100 Chieti, Italy, e-mail: pconti@ unich.it 
mulate in the human arterial intima and adventitia during atherosclerosis and actively participate in plaque destabilization) are present [13]. It is well established that Th1 cell subset activation promotes inflammatory response and plays an important role in atherosclerosis [14]; while Th2 cells mediate both atherogenic and anti-atherogenic [and anti-inflammatory] effects by generating certain cytokines such as interleukins (IL), including IL-10, IL-4, IL-13 and IL-37 $[11,15]$. These interleukins inhibit the activation of macrophage and dendritic cells and therefore the over-expression of inflammatory cytokines. IL-38 is a member of the IL- 1 cytokine family which binds to IL-36 receptor (IL-36R) and also has anti-inflammatory properties exerting a protective effect in some autoimmune diseases [11].

Interestingly, oxidized LDL-specific Tregs not only reduce the initiation, but also the progression of atherosclerosis and plaque formation $[16,17]$. These effects can also be mediated by statin drugs, which regulate $\mathrm{T}_{\mathrm{H}} 1 / \mathrm{T}_{\mathrm{H}} 2$ imbalance both in vitro and in vivo [18]. Tregs and their main subsets $\mathrm{CD}^{+} \mathrm{CD} 25^{+}$, Foxp3 ${ }^{+}$, T cells (natural Tregs) are crucial in mediating immune homeostasis and promoting the establishment and maintenance of peripheral tolerance [18]. They are also associated with an increased risk of atherosclerosis, diabetes [19, 20], fatty liver disease, autoimmune diseases and cancer [20].

TH17 cells contribute to the atherogenesis process and are involved in plaque formation [15]. In addition, vascular arterial dendritic cells which are similar to Langerhans cells of the skin, are involved in atherosclerotic lesions [21]. CD $11 b^{+}$cells are myeloid cells in early differential stages that include dendritic cells, immature macrophages, and granulocytes [22]. CD11 $\mathrm{b}^{+}$cells are critical to atherogenesis but their inhibition does not reduce atherogenic plaque $[22,23]$.

The blood vessel reaction is one of the most striking events in inflammation. It consists of vasodilation and vasoconstriction as well as in an increase in vascular permeability in the inflamed area [24]. The impairment of vasoreactivity found with edema, resembles the impairment of vasoreactivity found in pre-arteriosclerotic and arteriosclerotic animal models [24]. In arteriosclerosis, injury to the vessel wall leads to intimal smooth muscle cell proliferation and luminal occlusion [25, 26]. Low-density lipoprotein (LDL) remains the most important risk factor for this inflammatory disease process. Studies on the cell culture indicate that LDL has numerous direct actions on vascular smooth muscle cells (VSMC) [26]. One action is to decrease VSMC prostacyclin production, and down-regulation of cyclooxygenase expression [27, 28]. Apoptosis of endothelial cells can occur on exposure to circulating factors and inflammatory cells, leading to disruption of endothelial glycocalyx monolayer integrity [29]. In fact, endothelial cell apoptosis contributes to the pathogenesis of atherosclerosis and other vascular and circulatory disorders. Many pathogenic factors may promote apoptosis in endothelium, and these include oxidized LDL (oxLDL) and certain cytokines such as IL-1 and TNF [30]. OxLDL provokes a delayed, but sustained, increase in intracellular calcium in endothelial cells, which causes cell death, an effect that can be reversed by preventing the calcium increase [30]. Some authors reported that oxLDL provokes depletion of cholesterol in endothelial cells via the scavenger receptor CD36, leading to ineffective e-NOS targeting to cholesterol and an attenuated capacity to activate the enzyme [31, 32].

Macrophages have two sub-populations: M1 and M2. Both take part in the atherosclerotic process, although with opposite roles [33]. M1 macrophages generate high levels of IL-12, IL-23, IL-6, IL-1, and TNF; while activated M2 macrophages produce IL-4, IL-13, and IL-10 which can deactivate M1 macrophages, and all contribute to atherogenesis [34].

TNF is an important pro-inflammatory cytokine, capable of classical activation of macrophages to the M1 phenotype, thereby inducing the production of other pro-inflammatory Th1 cytokines.

We know that IL-1 induces TNF and activates endothelial cell apoptosis along with growth factor deprivation [35]. Platelet-derived growth factor (PDGF) is another cytokine, generated by a number of cell types, including macrophages, platelets, endothelial cells and smooth muscle cells, which induces both smooth muscle migration and proliferation [36]. It also participates in atherogenesis.

Levels of PDGF mRNA in intact arteries in vivo are very low, while certain stimuli, such as hypercholesterolemic serum, may activate the synthesis and release of inflammatory cytokines, including PDGF [37]. It is possible that high levels of pro-inflammatory cytokines lead to imbalance of effector/regulatory T-cells in atherosclerosis and other chronic diseases, forming an attractive new immunotherapy [38].

A monoclonal antibody that targets inflammatory cytokines, such as IL-1 and TNF- $\alpha$ receptors, may alleviate symptoms in patients with inflammatory diseases such as arteriosclerosis [38]. Expression and generation of IL-8 and IL- 6 are also correlated with plaque formation in human atherosclerosis, an effect that is likely mediated by local activation of perivascular mast cells, since these cells increase vascular leakage, induce plaque hemorrhage, provoke macrophage apoptosis, and result in leukocyte infiltration through the CXCR2, an IL-8 receptor [39, 40]. LDL and $\beta$-very low density lipoprotein ( $\beta$-VLDL) have an effect on the incidence of coronary heart disease and together are, with others, an important risk factor [41]. Lipids, mostly in form of cholesterol and cholesteryl ester, are a serious problem for the circulatory system, heart failure [41], and the overload cholesterol in macrophage foam cells of the arterial wall [42]. This leads to the development of atherosclerotic plaque and accumulation of cholesteryl esters in the cytoplasm of macrophages, trans- 
forming them into foam cells $[43,44]$. However, the precise mechanisms by which the increased levels of lipids induce atherosclerotic lesions are still unclear.

Mast cells participate in both innate and adaptive immunity and play an important role in maintaining a healthy physiology in wound healing and angiogenesis [45]. Considerable evidence indicates that mast cells play a key role in inflammation, and their products modulate inflammatory mediator production [45]. Upon activation, mast cells release a broad spectrum of pro-inflammatory cytokines, growth factors, vasoactive substances, and proteolytic enzymes [46]. Human mast cells are derived from a common myeloid progenitor and have high-affinity IgE receptors (FceRI) [47]. They are predominantly localized in mucosal and connective tissues and are distributed along blood vessels [48, 49]. Activated mast cells can have detrimental effects on their immediate surroundings in the vessel wall, provoking matrix degradation, apoptosis and enhancement as well as recruitment of inflammatory cells, which actively contributes to atherosclerosis and plaque formation [ 47 , $48,50]$. In recent years, the mast cell has been implicated in inflammatory disease processes, such as cardiovascular disease and atherosclerotic plaque progression [51-53]. Mast cells are the major effector cell in inflammation, allergy and asthma [54]. They have been shown to accumulate in atherosclerotic plaque and in the perivascular tissue during this disease [55]. Their numbers increase within the arterial wall during atherosclerosis, and they are found in the human arterial intima and adventitia during atherosclerotic plaque progression, as well as participating in plaque destabilization [56]. Although vascular mast cells are rare, they are found within lesions of atherosclerotic plaques, especially in the location of rupture-prone shoulder regions [56]. Mast cells generate proteases such as tryptase and chymase [57]. The activation of these enzymes can cause intra-plaque hemorrhage, macrophage and endothelial cell apoptosis, vascular leakage, and cytokine/chemokine production, which lead to the recruitment of leukocytes to the plaque [58-60]. Mast cells release angiogenic compounds, which induce not only growth of microvessels, but also result in leakiness and rupture of the fragile neo-vessels [48]. This may result in intraplaque hemorrhage. Mast cells are present in human arterial intima where they can degranulate after stimulation and secrete chymase, which inhibits HDL apolipoprotein and may retard the efflux of cellular cholesterol. In fact, it has been reported by Lee and colleagues that mast cell chymase provokes the degradation and lowers the levels of ApoA-I, the main HDL apolipoprotein, altering lipid metabolism [61].

MCs express toll like receptors (TLR) including TLR-9 and TLR-3, which can be activated by infections and lead to the generation of several cytokines and chemokines such as TNF, IFN- $\gamma$, IL-6, and IL-8 [62]. They accumulate in the stroma of a number of inflamed tissues in response to locally produced chemotactic factors for monocytes/mast cells, such as RANTES and MCP-1 [63, 64]. Since mast cells express CCR3 receptor, it is possible that they are recruited in the atherosclerotic plaque by certain chemokines, including eotaxin [63]. The inhibition of this receptor prevents mast cell migration into the inflammatory site. In 1997, we reported for the first time that injection of chemokines RANTES and MCP-1 under the rat skin produced inflammation and mast cell recruitment [63-66]. It was also reported that MCP-1 and macrophage accumulation in the atheromatous plaque may play a pivotal role in restenosis after percutaneous trans-luminal coronary angioplasty and induce luminal renarrowing, at least in part, by inducing $\mathrm{O}_{2}^{-}$release in monocytes [67]. Further understanding of the mechanism[s] by which MCP-1 is generated and acts after arterial injury may provide insights into therapies to limit the progression of atherosclerosis and restenosis.

IL-1 and TNF are macrophage products capable of inducing increased mast cell adhesion, along with macrophages, which also produce chemotactic factors such as LTB4 [68, 69]. Chemokines and C5a for leukocytes, including $\mathrm{T}$ cells, participate in a process that may form an amplification mechanism for the recruitment of further immune cells into atheromatous plaque [69].

Mast cells increase local inflammation with an augmentation of immune cells such as T lymphocytes and macrophages, which increase atherosclerosis, an effect abolished in mast cell-deficient $\mathrm{Kit}^{\mathrm{W}-\mathrm{sh} / \mathrm{W}-\mathrm{sh}} \mathrm{Ldlr}^{-/-}$mice [70].

The inhibition of mast cell diminishes the generation of the inflammatory products, and as a result, atherosclerosis.

Below we report a few examples of the inhibitory mast cell compounds:

Syk is an inflammatory receptor located in immune cells, including mast cells, which mediates atherosclerosis $[70,71]$. Therefore, this receptor can be a potential target not only for the treatment of allergic reactions, but also to alleviate atherosclerotic and cardiovascular diseases [71].

HYPERLIPIDEMIA $\rightarrow$ ENDOTHELIAL AND MAST

CELLS $\rightarrow$ CYTOKINES/CHEMOKINES $\rightarrow$ MONOCYTES ADHESION $\rightarrow$ FOAM

CELLS $\rightarrow$ ATHEROMATOUS PLAQUE

Fig. 1. Schematic representation of a possible sequence of events in atherogenesis 
Kit ligand is a stem cell factor for mast cell growth and differentiation; the inhibition of this factor may be a good and valid approach to prevent inflammatory reactions mediated by mast cells, including atherosclerosis [72, 73]. Therefore, inhibition of mast cell through kit ligand activation may be of interest for future therapeutic interventions.

CD40L: mast cells, along with other immune/inflammatory cells also express CD40L, which binds its receptor CD40 and initiates the inflammatory process contributing to the pathophysiology of atherosclerosis [73]. Therefore, the inhibition of CD40L levels can have a beneficial effect on patients with atherosclerosis and myocardial infarction, making this ligand a novel target for the treatment of atherosclerosis and other inflammatory diseases.

STATINS: It is interesting that statins can inhibit the levels of IgE and IgG-oxLDL. This effect may provide a mechanism for statins as potential anti-atherosclerotic drugs [72-74].

Statins are a class of cholesterol-lowering drugs and it has been reported that they exert beneficial effects in atherosclerosis along with TH2 cytokines such as IL-10 and IL-4, which have anti-atherogenic properties [72]. In addition, statins, like simvastatin, are capable of stabilizing atherosclerotic plaques by increasing Treg cells [73].

Statins influence the generation of cytokines released by $\mathrm{TH} 2$ cells, and have an anti-atherogenic property; therefore, they have beneficial effects on atherosclerosis. Moreover, these drugs suppress the secretion of pro-inflammatory cytokines, including IL-1 $\beta$ and IL-6, but not TNF [74]. $e$ NOS plays a crucial role in mediating this anti-inflammatory action [75]. Its over-expression reduces post-ischemic hyperpermeability of coronary microcirculation and the reperfusion injury of the heart in an experimental animal model [75-77]. These results support human studies suggesting that statins inhibit inflammatory cells in the atherosclerotic plaque [78, 79]. Monitoring atherosclerosis is very important and therapeutic interventions at different sites of the inflammatory process should be considered. Therapeutic targets could include statins, anti-cytokine/ chemokine receptors, anti-growth factors, anti-transcription factors, specific monoclonal antibodies, and others. In addition, inhibition of mast cell activation may be of interest for future therapeutic interventions. One of the protective actions of statins is mediated by nitric oxide (NO) [79, 80]. Statins up-regulate eNOS, which can activate NF- $\kappa$ B and cause its translocation to the cell nucleus. We previously reported that statin treatment in atherosclerosis was able to reduce plaque, microcirculatory dysfunction and necrotic cell death, thereby ameliorating tissue ultrastructure damage and provoking endothelial protection, probably by inducing endothelial nitric oxide synthase (eNOS) over-expression and reducing hyperpermeability [79-81]. Moreover, addition of vitamin D to an atherosclerotic animal model resulted in an increase in NO, demonstrating that vitamin D has anti-atherosclerosis effects [82].
However, physical activity, diet, and stress reduction can successfully modify cardiovascular risk factors [83].

Therefore, future novel therapeutic targets may be the inhibition of specific pathways of atherosclerosis-induced mast cell activation with some anti-receptors, stabilizers, Tregs or natural compounds such as flavonoids [luteolin or quercetin], which have been found to have anti-oxidant and beneficial effects in reducing inflammatory diseases by inhibiting the release of histamine, leukotrienes, prostaglandin D2, IL-6, IL-8, TNF and tryptase release from human cultured MCs [84].

Accumulation of lipids in vascular endothelium activates mast cells to produce cytokines and chemokines, which recruit macrophages [85]. On the other hand, mast cells augment inflammatory response by secreting the vascular endothelial growth factor, a key cytokine that mediates angiogenesis and the inflammatory response. The concepts expressed in this article support the importance of inflammatory mechanisms in the pathogenesis of atherosclerosis and suggest a possible role for mast cells, in the control of VSMC proliferation and atheromatous plaque $[85,86]$.

In conclusion, MC activation certainly participates in the process of the plaque progression and destabilization by an increased lipid uptake, leukocyte influx, apoptosis, matrix degradation, and intraplaque hemorrhage [87- 89].

These studies are not only of interest for the basic research field, but may also have relevance for clinical atherosclerosis and cardiovascular diseases [90].

However, the precise mechanism by which the increased levels of cholesterol induce the plaque and the progression of atherosclerosis, including the precise pathogenesis of such a widespread disorder, is still far from established. Taken together, these findings might provide new insights to explore potential targets for immune therapeutic intervention in atherosclerosis. Further studies are needed to determine the role of mast cells in the development of atherosclerosis and in general in the inflammatory response. Identifying specific intervention strategies are urgently needed, because they may provide novel therapies in the prevention of atherosclerosis including the dynamic of plaque formation.

The authors declare no conflict of interest.

*GRANT: During the preparation of this work, support was received from the Ministero Italiano dell'Università della Pubblica Istruzione, 2014.

\section{References}

1. Rock FL, Hardiman G, Timans JC, et al. (1998): A family of human receptors structurally related to Drosophila Toll. Proc Natl Acad Sci U S A 95: 588-593. 
2. Rosenfeld ME (2013): Inflammation and atherosclerosis: direct versus indirect mechanisms. Curr Opin Pharmacol 13: 154-160.

3. Parks BW, Lusis AJ (2013): Macrophage accumulation in atherosclerosis. N Engl J Med 369: 2352-2353.

4. Spirig R, Tsui J, Shaw S (2012): The emerging role of tlr and innate immunity in cardiovascular disease. Cardiol Res Pract 18: 1394.

5. O'Neal RM, Still WJ (1962): Pathogenesis of atherosclerosis. Fed Proc 21: 12-14.

6. Gerrity RG, Naito HK (1980): Ultrastructural identification of monocyte-derived foam cells in fatty streak lesions. Artery 8: 208-214.

7. Zhong Y, Wang X, Ji Q, et al. (2012): CD4+LAP + and CD4 $+\mathrm{CD} 25+$ Foxp3 + regulatory $\mathrm{T}$ cells induced by nasal oxidized low-density lipoprotein suppress effector $\mathrm{T}$ cells response and attenuate atherosclerosis in ApoE-/- mice. J Clin Immunol 32: 1104-1117.

8. Di Pietro M, De Santis F, Schiavoni G, et al. (2013): Resveratrol in Chlamydia pneumoniae-induced foam cell formation and interleukin-17A synthesis. J Biol Regul Homeost Agents 27: 509-518.

9. Medzhitov R (2007): Recognition of microorganisms and activation of the immune response. Nature 18: 819-826.

10. Meier P, Meier R, Blanc E (2008): Influence of CD4+/ $\mathrm{CD} 25+$ regulatory $\mathrm{T}$ cells on atherogenesis in patients with end-stage kidney disease. Expert Rev Cardiovasc Ther 6: 987-997.

11. Garlanda C, Dinarello CA, Mantovani A (2013): The interleukin-1 family: back to the future. Immunity 39: 1003-1018.

12. Chen Y, Jian Y, Liu M, et al. (2014): Gr- $1^{*} \mathrm{CD} 11^{\text {b* }}$ immature myeloid cells (IMC) promote resistance of pro-inflammatory $\mathrm{T}$ cells to suppression by regulatory $\mathrm{T}$ cells in atherosclerotic Apo E-deficient mice. PLoS One 9: e108620

13. Liu DQ, Li LM, Guo YL, et al. (2008): Signal regulatory protein alpha negatively regulates beta2 integrin-mediated monocyte adhesion, transendothelial migration and phagocytosis.

14. PLoS One. 29: e3291.

15. Mallat Z, Tedgui A. (2004): Immunomodulation to combat atherosclerosis: the potential role of immune regulatory cells. Expert Opin Biol Ther 9: 1387-1393.

16. Norata GD, Marchesi P, Passamonti S, et al. (2007): Anti-inflammatory and anti-atherogenic effects of cathechin, caffeic acid and trans-resveratrol in apolipoprotein $\mathrm{E}$ deficient mice. Atherosclerosis 191: 265-271.

17. Galkina E, Ley K. (2009): Immune and inflammatory mechanisms of atherosclerosis. Annu Rev Immunol 27: 165-197.

18. Li M, Lin J, Wang Z, He S, Ma X, Li D (2010): Oxidized low-density lipoprotein-induced proinflammatory cytokine response in macrophages are suppressed by CD4CD25(+) Foxp3(+) regulatory $\mathrm{T}$ cells through downregulating toll like receptor 2-mediated activation of NF-kappaB. Cell Physiol Biochem 25: 649-656.

19. Meng X, Zhang K, Li J, et al. (2012): Statins induce the accumulation of regulatory $\mathrm{T}$ cells in atherosclerotic plaque. Mol Med 18:598-605.

20. Goldstein JD, Pérol L, Zaragoza B (2013): Role of cytokines in thymus- versus peripherally derived-regulatory $\mathrm{T}$ cell differentiation and function. Front Immunol 19: 155.

21. Iantorno M, Campia U, Di Daniele N, et al. (2014): Gut hormones and endothelial dysfunction in patients with obesity and diabetes. Int J Immunopathol Pharmacol 27433-27436.
22. Pucino V, De Rosa V, Procaccini C, et al. (2014): Regulatory $\mathrm{T}$ cells, leptin and angiogenesis. Chem Immunol Allergy 99: 155-169.

23. Bobryshev YV, Lord RS (1995): Ultrastructural recognition of cells with dendritic cell morphology in human aortic intima. Contacting interactions of Vascular Dendritic Cells in athero-resistant and athero-prone areas of the normal aorta. Arch Histol Cytol 58: 307-322.

24. Stoneman V, Braganza D, vvg N, et al. (2007): Monocyte/ macrophage suppression in $\mathrm{CD} 11 \mathrm{~b}$ diphtheria toxin receptor transgenic mice differentially affects atherogenesis and established plaques. Circ Res 100: 884-893.

25. Issekutz AC, Movat HZ (1982): The effect of vasodilator prostaglandins on polymorphonuclear leukocyte infiltration and vascular injury. Am J Pathol 107:300-9.

26. Pedersen SF, Thrysře SA, Paaske WP, et al. (2011): Determination of edema in porcine coronary arteries by $\mathrm{T} 2$ weighted cardiovascular magnetic resonance. J Cardiovasc Magn Reson 13: 52

27. Hancock WW, Adams DH, Wyner LR, et al. (1994): CD4+ mononuclear cells induce cytokine expression, vascular smooth muscle cell proliferation, and arterial occlusion after endothelial injury. Am J Pathol 145: 1008-1014.

28. Inagami T (1994): Atrial natriuretic factor as a volume regulator. J Clin Pharmacol 34: 424-426.

29. Wang M, Zukas AM, Hui Y, et al. (2006): Eletion of microsomal prostaglandin E synthase-1 augments prostacyclin and retards atherogenesis. See comment in PubMed Commons belowProc Natl Acad Sci U S A 103: 14507-14512.

30. Schouten M, Wiersinga WJ, Levi M, et al (2008): Inflammation, endothelium, and coagulation in sepsis. J Leukoc Biol 83:536-45.

31. Shin HK, Kim YK, Lee JH, Hong KW (2004): Remnant lipoprotein particles induce apoptosis in endothelial cells by $\mathrm{NAD}(\mathrm{P}) \mathrm{H}$ oxidase-mediated production of superoxide and cytokines via lectin-like oxidized low-density lipoprotein receptor-1 activation: prevention by cilostazol. Circulation 109: $1022-1028$.

32. Muller C, Salvayre R, Nčgre-Salvayre A, et al (2011): HDLs inhibit endoplasmic reticulum stress and autophagic response induced by oxidized LDLs. Cell Death Differ 18: 817-828.

33. Mineo C, Shaul PW (2012): Novel biological functions of high-density lipoprotein cholesterol. Circ Res 111: 10791090.

34. da Rocha RF, De Bastiani MA, Klamt F (2014): Bioinformatics approach to evaluate differential gene expression of M1/ M2 macrophage phenotypes and antioxidant genes in atherosclerosis. Cell Biochem Biophys 70: 831-839.

35. El Hadri K, Mahmood DF, Couchie D, et al (2012): Thioredoxin-1 promotes anti-inflammatory macrophages of the M2 phenotype and antagonizes atherosclerosis. Arterioscler Thromb Vasc Biol 32:1445-1452.

36. Madge LA, Pober JS A (2000): A phosphatidylinositol 3-kinase/Akt pathway, activated by tumor necrosis factor or interleukin-1, inhibits apoptosis but does not activate NFkappaB in human endothelial cells. J Biol Chem 275: 15458-15465.

37. Hughes AD, Clunn GF, Refson J, et al. (1996): Platelet-derived growth factor (PDGF): actions and mechanisms in vascular smooth muscle. Gen Pharmacol 27: 1079-1089.

38. Alexandru N, Popov D, Dragan E, et al. (2013): Circulating endothelial progenitor cell and platelet microparticle impact on platelet activation in hypertension associated with hypercholesterolemia. PLoS One 8: e52058. 
39. Foks AC, Frodermann V, ter Borg M, et al. (2011): Differential effects of regulatory $\mathrm{T}$ cells on the initiation and regression of atherosclerosis. Atherosclerosis 21853-21860.

40. Yassin LM, Rojas M, Ramírez LA, et al. (2010): Monocyte activation by apoptotic cells removal in systemic lupus erythematosus patients. Cell Immunol 266: 52-60.

41. Li H, Huang S, Wang S, et al. (2013):Targeting annexin A7 by a small molecule suppressed the activity of phosphatidylcholine-specific phospholipase $\mathrm{C}$ in vascular endothelial cells and inhibited atherosclerosis in apolipoprotein $\mathrm{E}^{-/}$mice. Cell Death Dis 19: e806.

42. Ren J, Grundy SM, Liu J, et al. (2010): Long-term coronary heart disease risk associated with very-low-density lipoprotein cholesterol in Chinese: the results of a 15-Year Chinese Multi-Provincial Cohort Study (CMCS). Atherosclerosis 211: 327-332.

43. Hoff HF, O'Neil J (1988): Extracts of human atherosclerotic lesions can modify low density lipoproteins leading to enhanced uptake by macrophages. Atherosclerosis 70: 29-41.

44. Falcone C, Bozzini S, Matrone B, et al. (2013): RAGE gene polymorphism in heart failure patients with and without angiographic evidence of significant coronary atherosclerosis. Int J Immunopathol Pharmacol 26: 199-206.

45. Fukuda S, Horiuchi S, Tomita K, et al (1986): Acetylated low-density lipoprotein is endocytosed through coated pits by rat peritoneal macrophages. Virchows Arch B Cell Pathol Incl Mol Pathol 52: 1-13.

46. Kritas SK, Caraffa A, Antinolfi P, et al. (2014):IgE Generation and mast cell activation. Eur J Inflamm 12: 21-25.

47. Kritas SK, Saggini A, Cerulli G, et al. (2014): Relationship between serotonin and mast cells: inhibitory effect of anti-serotonin. J Biol Regul Homeost Agents 28: 377-380.

48. Kritas SK, Saggini A, Cerulli G, et al. (2014): Neuropeptide NGF mediates neuro-immune response and inflammation through mast cell activation. J Biol Regul Homeost Agents 28: 177-181.

49. Bot I, Shi GP, Kovanen PT (2015): Mast cells as effectors in atherosclerosis. Arterioscler Thromb Vasc Biol 35: 265-271.

50. Kritas SK, Saggini A, Varvara G, et al (2013): Impact of mast cells on the skin. Int J Immunopathol Pharmacol 26: 855-859.

51. Kritas SK, Saggini A, Varvara G, et al. (2013): Mast cell involvement in rheumatoid arthritis. J Biol Regul Homeost Agents 27: 655-660.

52. Artenjak A, Koželj M, Lakota K, et al. (2013): High avidity anti- $\beta 2$-glycoprotein I antibodies activate human coronary artery endothelial cells and trigger peripheral blood mononuclear cell migration. Eur J Inflamm 11: 385-396.

53. Shaik-Dasthagirisaheb YB, Varvara G, Murmura G, et al (2013): Vascular endothelial growth factor (VEGF), mast cells and inflammation. Int J Immunopathol Pharmacol 26: 327-335.

54. Conti P, Varvara G, Murmura G, et al. (2013): Comparison of beneficial actions of non-steroidal anti-inflammatory drugs to flavonoids. J Biol Regul Homeost Agents 27: 1-7.

55. Bot I, de Jager SC, Bot M, et al. (2010): The neuropeptide substance $\mathrm{P}$ mediates adventitial mast cell activation and induces intraplaque hemorrhage in advanced atherosclerosis. Circ Res 106: 89-92.

56. den Dekker WK, Tempel D, Bot I, et al. (2012): Mast cells induce vascular smooth muscle cell apoptosis via a toll-like receptor 4 activation pathway. Arterioscler Thromb Vasc Biol 32: 1960-1969.
57. Willems S, Vink A, Bot I, et al (2013): Mast cells in human carotid atherosclerotic plaques are associated with intraplaque microvessel density and the occurrence of future cardiovascular events. Eur Heart J 34: 3699-3706.

58. Frydas S, Varvara G, Murmura G, et al (2013): Impact of capsaicin on mast cell inflammation. Int J Immunopathol Pharmacol 26: 597-600.

59. Speranza L, Pantalone A, Saggini R, et al. (2013): Inhibitor effect of antioxidant flavonoids quercitin, and capsaicin in mast cell inflammation Eur J Inflamm 11: 353-357.

60. Galkina E, Ley K (2007): Leukocyte influx in atherosclerosis. Curr Drug Targets 8: 1239-1248.

61. Kritas SK, Saggini A, Cerulli G, et al (2014): Interrelationship between IL-3 and mast cells. J Biol Regul Homeost Agents 28: 17-21.

62. Lee M, Calabresi L, Chiesa G, (2002): Mast cell chymase degrades apoE and apoA-II in apoA-I-knockout mouse plasma and reduces its ability to promote cellular cholesterol efflux. Arterioscler Thromb Vasc Biol 22: 1475-1481.

63. Raicevic G, Rouas R, Najar M, et al. (2010): Inflammation modifies the pattern and the function of Toll-like receptors expressed by human mesenchymal stromal cells. Hum Immunol 71: 235-244.

64. Taub DD, Proost P, Murphy WJ, et al (1995): Monocyte chemotactic protein-1 (MCP-1), -2, and -3 are chemotactic for human T lymphocytes. J Clin Invest 95: 1370-1376.

65. Renne M, Conforti F, Camastra C, et al. (2014): Macrophage activation and patterns of inflammation in obese and nonobese women with breast carcinoma. Eur J Inflamm 12: 197200.

66. Haley KJ, Lilly CM, Yang JH, et al. (2000): Overexpression of eotaxin and the CCR3 receptor in human atherosclerosis: using genomic technology to identify a potential novel pathway of vascular inflammation. Circulation 102: 2185-2189.

67. Conti P, Pang X, Boucher W, et al. (1997): Impact of Rantes and MCP-1 chemokines on in vivo basophilic cell recruitment in rat skin injection model and their role in modifying the protein and mRNA levels for histidine decarboxylase. Blood 89: 4120-4127.

68. Cipollone F, Marini M, Fazia M, et al. (2001): Elevated circulating levels of monocyte chemoattractant protein- 1 in patients with restenosis after coronary angioplasty. Arterioscler Thromb Vasc Biol 21: 327-334.

69. Oliveira SH, Costa CH, Ferreira SH, et al. (2002): Sephadex induces eosinophil migration to the rat and mouse peritoneal cavity: involvement of mast cells, LTB4, TNF-alpha, IL-8 and PAF. Inflamm Res 51: 144-153.

70. McNeill E, Iqbal AJ, Patel J, et al. (2014): Contrasting in vitro vs. in vivo effects of a cell membrane-specific CC-chemokine binding protein on macrophage chemotaxis. J Mol Med (Berl) 92: 1169-1178.

71. Sun J, Sukhova GK, Wolters PJ, et al. (2007): Mast cells promote atherosclerosis by releasing proinflammatory cytokines. Nat Med 13: 719-724.

72. Baumruker T, Csonga R, Pursch E, et al. (2003): Activation of mast cells by incorporation of cholesterol into rafts. Int Immunol 15: 1207-1218.

73. Michel JB (2003): Anoikis in the cardiovascular system: known and unknown extracellular mediators. Arterioscler Thromb Vasc Biol 23: 2146-2154.

74. Grewal IS, Flavell RA (1998): CD40 and CD154 in cell-mediated immunity. Annu Rev Immunol 16: 111-135. 
75. Willems S, van der Velden D, Quax PH, et al (2014): Circulating immunoglobulins are not associated with intraplaque mast cell number and other vulnerable plaque characteristics in patients with carotid artery stenosis. PLoS One 9: e88984.

76. Montero MT, Hernández O, Suárez Y, et al (2000): Hydroxymethylglutaryl-coenzyme A reductase inhibition stimulates caspase-1 activity and Th1-cytokine release in peripheral blood mononuclear cells. Atherosclerosis 153: 303-313.

77. Matusik P, Guzik B, Weber C, Guzik TJ (2012): Do we know enough about the immune pathogenesis of acute coronary syndromes to improve clinical practice? Thromb Haemost 108: 443-456.

78. Zanin V, Marcuzzi A, Kleiner G, et al (2013): Lovastatin dose-dependently potentiates the pro-inflammatory activity of lipopolysaccharide both in vitro and in vivo. J Cardiovasc Transl Res 6: 981-988.

79. Barsotti A, Di Napoli P, Taccardi AA, et al. (2001): MK954 (losartan potassium) exerts endothelial protective effects against reperfusion injury: evidence of an e-NOS mRNA overexpression after global ischemia. Atherosclerosis 155: 53-59.

80. Vaughan CJ, Gotto AM Jr, Basson CT (2000): The evolving role of statins in the management of atherosclerosis. J Am Coll Cardiol 35: 1-10.

81. Rajanikant GK, Zemke D, Kassab M, et al. (2007): The therapeutic potential of statins in neurological disorders. Curr Med Chem 14: 103-112.

82. Weng Z, Zhang B, Asadi S, et al. (2012): Quercetin is more effective than cromolyn in blocking human mast cell cytokine release and inhibits contact dermatitis and photosensitivity in humans. PLoS One 7: e33805.

83. Malek HA, Shata A (2014): Effect of a high dose of vitamin $\mathrm{D}$ on a rabbit model of atherosclerosis. Int J Immunopathol Pharmacol 27: 195-201.

84. De Liso F, Bonara P, Vigna L, et al (2013): Oxidative stress and low-grade inflammatory status as cardiometabolic risk factors in Italian occupational overweight/obese subjects. Eur J Inflamm 11: 789-796.

85. Rickard A, Portell C, Kell PJ, et al. (2005): Protease-activated receptor stimulation activates a $\mathrm{Ca} 2+$-independent phospholipase A2 in bladder microvascular endothelial cells. Am J Physiol Renal Physiol 288: F714-F721.

86. Porreca E, Di Febbo C, Di Sciullo A, et al. (1996): Cysteinyl leukotriene D4 induced vascular smooth muscle cell proliferation: a possible role in myointimal hyperplasia. Thromb Haemost 76: 99-104.

87. Zardi EM, Sambataro G, Basta F, et al. (2014): Subclinical carotid atherosclerosis in elderly patients with primary Sjögren syndrome: a duplex Doppler sonographic study. Int J Immunopathol Pharmacol 27: 645-651.

88. Shaik-Dasthagirisaheb YB, Varvara G, Murmura G, et al. (2013): Inhibitor effect of antioxidant flavonoids quercitin, and capsaicin in mast cell inflammation. Eur J Inflamm 11: 353-357.

89. Yoshino Y, Kohara K, Abe M, et al (2014): Missense variants of the alanine: glyoxylate aminotransferase 2 gene correlated with carotid atherosclerosis in the Japanese population. J Biol Regul Homeost Agents 28: 605-614.

90. Keum JS, Jaffa MA, Luttrell LM, et al (2014): Novel mechanism of plasma prekallikrein (PK) activation by vascular smooth muscle cells: evidence of the presence of PK activator. J Biol Regul Homeost Agents 28: 587-603. 